\title{
Dispersion-managed soliton in optical fibers with zero average dispersion
}

\author{
P. M. Lushnikov \\ Theoretical Division, Los Alamos National Laboratory, MS-B284, Los Alamos, New Mexico 87545, and \\ Landau Institute for Theoretical Physics, Kosygin Street 2, Moscow 117334, Russia
}

Received March 10, 2000

A dispersion-managed optical system with stepwise periodic variation of dispersion is studied in the framework of a path-averaged Gabitov-Turitsyn equation. The soliton solution is obtained by means of iterating the path-averaged equation. The dependence of soliton parameters on dispersion map strength is investigated, together with the oscillating tails of the soliton. (C) 2000 Optical Society of America

OCIS codes: $\quad 060.2330,060.5530,060.4370,190.5530,260.2030$.

A dispersion-managed (DM) system, ${ }^{1}$ which is a system with periodic variation of dispersion along an optical fiber, is one of the key components of development of ultrafast high-bit-rate optical communication lines. ${ }^{2-7}$ The main factor that limits bit-rate capacity is pulse broadening, which is due to chromatic dispersion of the optical fiber and can be overcome by periodic changes of the sign of the fiber's dispersion to create low (or even zero) path-averaged dispersion. Lossless propagation of an optical pulse in a DM fiber is described by a nonlinear Schrödinger equation with periodically varying dispersion $d(z)$ :

$$
i u_{z}+d(z) u_{t t}+|u|^{2} u=0,
$$

where $u$ is the envelope of the optical pulse, $z$ is the propagation distance, and all quantities are made dimensionless. Consider a two-step periodic dispersion map: $d(z)=\langle d\rangle+\tilde{d}(z)$, where $\tilde{d}(z)=d_{1}$ for $0<z+n L<L / 2$ and $\tilde{d}(z)=-d_{1}$ for $L / 2<z+n L<L ;\langle d\rangle$ is the path-averaged dispersion; $d_{1}$ is the amplitude of the dispersion variation; $L$ is a dispersion period; and $n$ is an arbitrary integer number. Equation (1) also describes pulse propagation in a fiber with losses compensated for by periodically placed amplifiers if the distance between amplifiers is much less than $L$.

Provided that the characteristic nonlinear length $Z_{\mathrm{NL}}$ of the pulse is large, i.e., $Z_{\mathrm{NL}}>>L$, where $Z_{\mathrm{NL}}=1 /|p|^{2}$ and $p$ is a typical pulse amplitude, Eq. (1) can be reduced to a path-averaged Gabitov-Turitsyn ${ }^{4}$ model:

$$
\begin{aligned}
i \hat{\psi}_{z}(\omega) & -\omega^{2}\langle d\rangle \hat{\psi}+\frac{1}{(2 \pi)^{2}} \int \frac{\sin s \omega_{1} \omega_{2}}{s \omega_{1} \omega_{2}} \hat{\psi}\left(\omega_{1}+\omega\right) \\
& \times \hat{\psi}\left(\omega_{2}+\omega\right) \hat{\psi}^{*}\left(\omega_{1}+\omega_{2}+\omega\right) \mathrm{d} \omega_{1} \mathrm{~d} \omega_{2}=0,
\end{aligned}
$$

where $s=d_{1} L / 2$ is the dispersion map strength, $\hat{\psi} \equiv$ $\hat{u} \exp \left[i \omega^{2} \int_{L / 4}^{z} \tilde{d}\left(z^{\prime}\right) \mathrm{d} z^{\prime}\right]$, and $\hat{\psi}(\omega)=\int_{-\infty}^{\infty} \psi(t) \exp (i \omega t) \mathrm{d} t$ is a Fourier component of $\psi$. The Gabitov-Turitsyn model is based on the assumption of slow variation of $\hat{\psi}$ as a function of $z$ on scales of the order of DM period $L$. This model is well supported by numerical simulations. ${ }^{8,9}$ Returning to $t$ space, one can get $^{9}$

$$
\begin{array}{r}
i \psi_{z}+\langle d\rangle \psi_{t t}-\frac{1}{2 \pi s} \int C i\left(\frac{t_{1} t_{2}}{s}\right) \psi\left(t_{1}+t\right) \psi\left(t_{2}+t\right) \\
\times \psi^{*}\left(t_{1}+t_{2}+t\right) \mathrm{d} t_{1} \mathrm{~d} t_{2}=0,
\end{array}
$$

where $C i(x)=\int_{\infty}^{x}(\cos x / x) \mathrm{d} x$ [note the difference in the definition of $C i(x)$ compared with that in Ref. 9]. It was found numerically ${ }^{3}$ that the Gaussian ansatz

$$
A_{\mathrm{Gauss}}=p \exp \left(-\frac{\beta}{2} t^{2}\right),
$$

where $p$ and $\beta$ are real constants, is a rather good approximation for the soliton solution $u=A(t) \exp (i \lambda z)$ ( $A$ is real) of Eq. (1) for small and moderate values of $t$ at space points $z=L / 4+n L$. Equation (4) is also a good approximation for the soliton solution $\psi=$ $A(t) \exp (i \lambda z)$ of Eq. (3). For the soliton solution Eq. (3) takes the form

$$
\begin{aligned}
-\lambda A+\langle d\rangle A_{t t}= & \frac{1}{2 \pi s} \int C i\left(\frac{t_{1} t_{2}}{s}\right) A\left(t_{1}+t\right) \\
& \times A\left(t_{2}+t\right) A\left(t_{1}+t_{2}+t\right) \mathrm{d} t_{1} \mathrm{~d} t_{2} .
\end{aligned}
$$

Based on this observation, the soliton solution of Eq. (1) was approximated in Refs. 10 and 11 by a Gaussian exponent multiplied by a sum consisting of a finite number of Hermite polynomials. Because an infinite set of these polynomials is complete in the space of a square-integrable function, $\psi$ can be expanded over this set. But for large enough $t$ such an approximation cannot be effective because of the highly oscillating tails ${ }^{9,10}$ of $A$ for $t \rightarrow \infty$, which require a large number of expansion terms to be taken into account to produce a reasonable approximation. The oscillating tails of $A$ are of great importance because they are responsible for the interaction of a sequence of solitons launched in optical fiber, which limits bit-rate capacity. ${ }^{6}$

Here the convergent set of approximate solutions of Eq. (5) is found by means of iterating that equation for $\langle d\rangle=0$. Zero iteration $A^{(0)}$ is given by Eq. (4). $-\lambda A^{(n)}$ is obtained by substitution of $A^{(n-1)}$ into the right-hand side of Eq. (5) for $n=1,2, \ldots$ The oscillating tails have already appeared at the first iteration. 
Simultaneously one can find the dependence of $\beta$ in Eq. (4) on dispersion map strength $s$.

Rewriting the kernel of Eq. (2) by means of the parametric integral

$$
\frac{\sin s \omega_{1} \omega_{2}}{s \omega_{1} \omega_{2}}=\frac{1}{2 s} \int_{-s}^{s} \exp \left(i s^{\prime} \omega_{1} \omega_{2}\right) \mathrm{d} s^{\prime}
$$

and using Eq. (4) as a zero iteration permit integration over time variables $t_{1}$ and $t_{2}$ in Eq. (3) explicitly yield the following expressions for first $A^{(1)}$, second $A^{(2)}$, etc. iterations:

$$
\begin{aligned}
& A^{(1)}=p^{(1)} \int_{-\tilde{s}}^{\tilde{s}} \frac{\exp \left\{-\left[(\beta / 2)\left(3 i+s^{\prime}\right) /\left(i+3 s^{\prime}\right)\right] t^{2}\right\}}{\sqrt{1-2 i s^{\prime}+3 s^{\prime 2}}} \mathrm{~d} s^{\prime} \\
& A^{(2)}=p^{(2)} \iiint \int_{-\tilde{s}}^{\tilde{s}} \frac{\exp \left(-q t^{2}\right)}{\sqrt{f_{1} f_{2} f_{3} g}} \mathrm{~d} s^{\prime} \mathrm{d} s_{1} \mathrm{~d} s_{2} \mathrm{~d} s_{3} \\
& A^{(3)}=\ldots
\end{aligned}
$$

where $\tilde{s}=\beta s$, integration limits for all variables are $[-\tilde{s}, \tilde{s}], f_{j}=1-2 i s_{j}+3 s_{j}{ }^{2}, b_{j}=\left(i+3 s_{j}\right) /[2 \beta(3 i+$ $\left.\left.s_{j}\right)\right], j=1,2,3, g=4\left[4 b_{1} b_{2} b_{3}-4 i b_{1} b_{2} s^{\prime}+\left(b_{1}+b_{2}+\right.\right.$ $\left.\left.b_{3}\right) s^{\prime 2}\right], q=\left[4\left(b_{1} b_{2}+b_{1} b_{3}+b_{2} b_{3}\right)+4 i s^{\prime} b_{3}+s^{\prime 2}\right] / g$, and $p^{(n)}$ is determined by the condition that $\left.A^{(n)}\right|_{t=0}=$ $p$ for all $n$. Note that instead of keeping $\left.A^{(n)}\right|_{t=0}$ constant for all iterations it is also possible to fix the integral $P_{0} \equiv \int\left|A^{(n)}\right|^{2} \mathrm{~d} t$ if one aims to get a soliton solution with some definite value of $P_{0}$. In principle any values of $\lambda$ and $\beta$ can be chosen for zero iteration, but it is most useful to choose them in such a way as to get $A^{(1)}$ as close as possible to $A^{(0)}$ to get a faster convergence of iterations to a true solution of Eq. (5). It is convenient to make series expansion $A^{(1)}$ in powers of $t^{2}$, which permits all terms of this expansion to be integrated explicitly. In particular,

$$
\begin{aligned}
p^{(1)}= & \sqrt{3} p /\left(\operatorname{arcsinh} \frac{3 \tilde{s}-i}{2}+\text { c.c. }\right), \\
\left.A_{t t}{ }^{(1)}\right|_{t=0}= & -p^{(1)} \frac{2 \beta}{3}\left(2 \sqrt{\frac{\tilde{s}+i}{3 \tilde{s}-i}}\right. \\
& \left.+\frac{\sqrt{3}}{6} \operatorname{arcsinh} \frac{3 \tilde{s}-i}{2}+\text { c.c. }\right)
\end{aligned}
$$

where c.c. means complex conjugation. Equating terms proportional $t^{2}$ in series expansions of $A^{(1)}$ and Eq. (4), one gets from Eqs. (8) a transcendental equation for $\tilde{s}$, which gives

$$
\tilde{s}=\beta s=2.393 \ldots
$$

and, with Eq. (5) with the same accuracy,

$$
\lambda=p^{2} \times 0.482 \ldots
$$

Figure 1 shows the results of the first four iterations obtained numerically for $p=1.7, d_{1}=500, L=0.01$, $s=2.5,\langle d\rangle=0$, and $\tilde{t}=t / \sqrt{s} ; \beta$ and $\lambda$ are given by Eqs. (9) and (10), respectively. It is enough to consider only positive values of $t$ because $A(t)$ is an even function of $t$. Note that when $\langle d\rangle=0$ Eq. (5) is invariant under two scaling transforms: $t \rightarrow \delta_{1} t, s \rightarrow \delta_{1}{ }^{2} s$,
$A(\tau) \rightarrow A\left(\tau / \delta_{1}\right)$ and $\lambda \rightarrow \delta_{2}{ }^{2} \lambda, A \rightarrow \delta_{2} A$, which allows the results of Fig. 1 to be extended to all values of system parameters $p$ and $s$ (of course it is sensible to consider only such values of $p$ and $s$ for which the condition $Z_{\mathrm{NL}} \gg L$ holds).

It can be seen from Fig. 1 that convergence of the iteration procedure to a soliton solution is very fast for small $t$ and becomes slower for larger $t$. For $|\tilde{t}| \lesssim 1.3$ the renormalization of $A^{(1)}$ by the next iteration is very small; in particular, for all $n=2,3, \ldots,\left.\left[A_{t t}{ }^{(n)} / A^{(n)}\right]\right|_{t=0}$ differs from $\beta$ determined by Eq. (9) by less than $2 \%$. For $|\tilde{t}| \leqslant 2.3$ a good approximation is given by a second iteration, for $|\tilde{t}| \lesssim 3.2$ by a third, etc. The sequence of iterations can be interpreted as the evolution of $A$ along some artificial coordinate $\tilde{z}$ and can indicate that the convergence of the solution of Eq. (3) during evolution along coordinate $z$ to the soliton solution [Eq. (5)] should also be slower for larger $t$ as is really observed in numerical experiments if $\left.\psi\right|_{z=0}$ is chosen in Gaussian form. ${ }^{2}$

Thus, provided that the form of the soliton is close to Gaussian for small and moderate $t$, the value of $\beta s$ is universal and is given by Eq. (9). Zakharov and Manakov $^{13}$ proposed another theory of a DM soliton in a strong DM limit based on the assumption that the typical width of an $A$ distribution is much less than $\sqrt{s}$; the present notation, this means that $\beta s \gg 1$. A priori one cannot exclude that a soliton solution with $\beta s \gg 1$ is also possible, in addition to the solution obtained here. But such a solution cannot be Gaussian-like, and, to the best of my knowledge, in all numerical experiments so far the value of $\beta s$ was of the order of unity (see, e.g., Refs. 3, 9, and 10). Note that the choice of $\beta s \gg 1$ for zero iteration results in the convergence of iteration sequence (7) to a Gaussian-like solution with $\beta$ given by Eq. (9), which can indicate that the solution of Eq. (3) for $\beta s \gg 1$ also converges for large $z$ to that Gaussian-like solution. Also, one cannot exclude the existence of a soliton with $\beta s \gg 1$ for $\langle d\rangle \neq 0$, which is outside the scope of this Letter.

Let us try to explore in more detail in what sense soliton solution $A$ is close to Gaussian. From Eqs. (7)-(9) one can get a series expansion (the approximate numerical value of $\tilde{s}$ is used here to avoid the need to write a cumbersome expression for every term of the expansion):

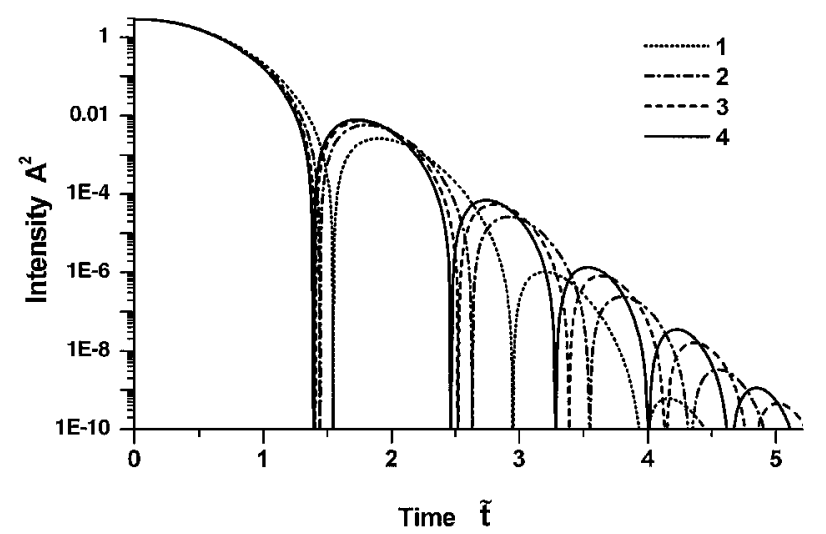

Fig. 1. Time dependence of first, second, third, and fourth iterations (curves 1, 2, 3, and 4, respectively). 


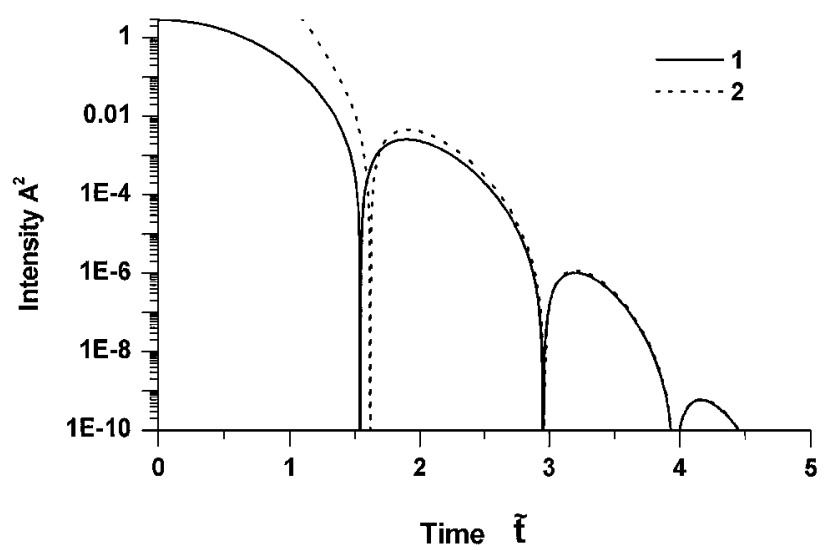

Fig. 2. First iteration $A^{(1)}(t)$ (curve 1) versus Eq. (12) (curve 2).

$$
\begin{aligned}
& A^{(1)} \exp \left(\frac{\beta t^{2}}{2}\right) / p \simeq 1-8.211 \times 10^{-2} \tilde{t}^{4}-2.654 \\
& \quad \times 10^{-2} \tilde{t}^{6}-4.383 \times 10^{-3} \tilde{t}^{8}-3.946 \times 10^{-4} \tilde{t}^{10}+\ldots
\end{aligned}
$$

One can conclude from this expansion that $A$ can be written as a multiplication of Gaussian exponent (4) on a slow function that changes significantly on scales $\tilde{t}^{2} \sim 3.5$. For this and larger scales there is no similarity between the soliton solution and Eq. (4). Thus the DM soliton is close to a Gaussian exponent only with an accuracy up to a numerically small parameter, which is of the order of $1 / 4$ (because the scale of the Gaussian exponent is $\beta t^{2} / 2=$ $\tilde{s} \tilde{t}^{2} / 2 \sim 1$ ), and there is no really small parameter that describes this closeness. Nevertheless the appearance of the numerically small parameter $1 / 4$ explains the success of the expansion of the DM soliton solution in Hermite polynomials, ${ }^{10,11}$ which is basically equivalent to expansion (11) in Taylor series.

For larger scales, $|\tilde{t}| \gtrsim 1.3$, the correct presentation of a DM soliton requires many terms of series expansion. However, one can get asymptotic behavior from Eqs. (7); e.g., the asymptote of the first iteration is given by

$$
\begin{aligned}
&\left.A\right|_{t \rightarrow \infty}=\frac{p^{(1)}}{16 \beta^{2} t^{2}} \exp \left(-\frac{\beta}{2} \frac{3 i+\tilde{s}}{i+3 \tilde{s}} t^{2}\right)\left(\frac{3 \tilde{s}+i}{\tilde{s}-i}\right)^{3 / 2} \\
& \quad \times\left[-4 i(\tilde{s}-i)+\frac{(3 \tilde{s}-5 i)(3 \tilde{s}+i)}{t^{2}}\right]+\text { c.c. }+O\left(\frac{1}{t^{6}}\right) .
\end{aligned}
$$

Figure 2 shows that Eq. (12) (curve 2) is a relatively good approximation of $A^{(1)}$ (curve 1 ) for $|\tilde{t}| \geqslant 1.6$. But of course to improve the accuracy of the soliton solution approximation it is necessary to get asymptotes of the next iterations. Another way is to use series expansion (11) instead of Eq. (4) as the zero iteration. That is, by keeping the first four terms of this expansion for zero iteration one can get a parametric integral similar to Eqs. (7), which gives a good approximation of $A^{(2)}$ in Eqs. (7) for $|\tilde{t}| \lesssim 3.5$. Detailed consideration of this approximation is outside the scope of this Letter.

The author thanks I. R. Gabitov and E. A. Kuznetsov for helpful discussions. Support for the this research was provided by the U.S. Department of Energy under contract W-7405-ENG-36, the Russian Foundation for Basic Research, and the program of government support for leading scientific schools. The author's e-mail address is lushnikov@cnls.lanl.gov.

\section{References}

1. C. Lin, H. Kogelnik, and L. G. Cohen, Opt. Lett. 5, 476 (1980).

2. M. Nakazawa and H. Kubota, Electron. Lett. 31, 216 (1995).

3. N. J. Smith, F. M. Knox, N. J. Doran, K. J. Blow, and I. Bennion, Electron. Lett. 32, 54 (1996).

4. I. Gabitov and S. K. Turitsyn, Opt. Lett. 21, 327 (1996); JETP Lett. 63, 861 (1996).

5. S. Kumar and A. Hasegawa, Opt. Lett. 22, 372 (1997).

6. P. V. Mamyshev and N. A. Mamysheva, Opt. Lett. 24, 1454 (1999).

7. L. F. Mollenauer, P. V. Mamyshev, J. Gripp, M. J. Neubelt, N. Mamysheva, L. Grüner-Nielsen, and T. Veng, Opt. Lett. 25, 704 (2000).

8. S. K. Turitsyn, N. J. Doran, J. H. B. Nijhof, V. K. Mezentsev, T. Schäfer, and W. Forysiak, in Optical Solitons: Theoretical Challenges and Industrial Perspectives, V. E. Zakharov and S. Wabnitz, eds. (Springer-Verlag, Berlin, 1999), p. 91.

9. M. J. Ablowitz and G. Biondini, Opt. Lett. 23, 1668 (1998).

10. S. K. Turitsyn and V. K. Mezentsev, JETP Lett. 67, 640 (1998); S. K. Turitsyn, Phys. Rev. E 58, 1256 (1998).

11. T. Lakoba and D. J. Kaup, Electron. Lett. 34, 1124 (1998).

12. V. K. Mezentsev, Institute of Automation and Electrometry, Russian Academy of Sciences, 630090, Novosibirsk, Russia (personal communication, 1999).

13. V. E. Zakharov, in Optical Solitons: Theoretical Challenges and Industrial Perspectives, V. E. Zakharov and S. Wabnitz, eds. (Springer-Verlag, Berlin, 1999), p. 73; V. E. Zakharov and S. V. Manakov, JETP Lett. 70, 578 (1999). 